\title{
Spirito dei tempi
}

\section{Spirit of the times}

Dipartimento di Scienze Biomediche, Chirurgiche e Odontoiatriche

Università degli Studi di Milano

via Beldiletto 1/3 Milano 20142

Italia

giovanni.lodi@unimi.it

tel. +390250319021

fax. +390250319041

"Io non credo alla letteratura" É la perentoria affermazione di un collega.

Mi chiedo cosa significhi. In bocca a chi di lavoro si prende cura della salute altrui, suona un po' come un pilota di aerei che affermi di non credere alle torri di controllo. Se non fosse che il pilota sconta personalmente eventuali errori, il medico o l'odontoiatra no. Sia come sia, mi sembra che posizioni come queste stiano diventando più comuni rispetto a qualche tempo fa, anche se con sfumature diverse, per cui l'oggetto della diffidenza può essere di volta in volta la evidence based medicine, le revisioni sistematiche o i trial randomizzati.

Affrontare i problemi di salute di chi si è affidato alle nostre cure basandoci sulla letteratura, o meglio, sulla ricerca clinica, non rappresenta un approccio elitario alla medicina, fatto di statistiche e prescrizioni calate dall'alto, che limita l'autonomia del medico. Al contrario, è un tentativo di offrire soluzioni basate su dati verificabili, ottenuti grazie a metodi condivisi, la cui alternativa è una medicina in cui trova cittadinanza l'aneddoto, la consuetudine, ciò che sembra funzionare.

Secondo l'Oxford English Dictionary, la parola dell'anno 2016 è post-verità. Neologismo diventato popolare in politica e comunicazione e che, a differenza della più familiare bugia, indica un atteggiamento per cui risulta irrilevante la differenza tra ciò che è vero e ciò che non lo è, tra fatti e opinioni. Come se la verità avesse perso posizioni nella gerarchia dei valori, a vantaggio di emozioni, sensazioni, pregiudizi o di quello che semplicemente appare plausibile. Ai tempi della post verità, chi non conosce può ottenere credito e legittimità quanto chi è competente.

Ecco, forse il collega prova ad adattarsi allo spirito dei tempi. O più semplicemente non ha idea di quello di cui parla, perché la letteratura non chiede di essere creduta, ma letta e compresa.

Buona lettura (appunto) 